\title{
Optimización de los parámetros tecnológicos para la obtención de piezas de latón por pulvimetalurgia ${ }^{(\cdot)}$
}

\author{
N. Krivij ${ }^{(*)}$, W. Suwardjo ${ }^{(*)}$ y A. Cores ${ }^{(* *)}$ \\ Resumen Se han producido polvos de latón 60 (40\% de Zn) y latón 80 (20\% de Zn) mediante la atomización \\ del metal fundido con agua a alta presión, en la planta de pulvimetalurgia del Centro de \\ Investigaciones Metalúrgicas (CIME) de La Habana. Se ha realizado la caracterización físico- \\ química y tecnológica y se han determinado los parámetros óptimos para conformar y sinterizar \\ piezas a partir de estos polvos. Las propiedades mecánicas de estas piezas pueden competir con las \\ de bronce y hierro, en aplicaciones para equipos electrodomésticos y en construcción.
}

Palabras clave: Piezas de latón. Pulvimetalurgia.

\section{Optimization of the technological parameters for obtaining brass details by powder metallurgy}

\begin{abstract}
Powders of brass $60(40 \% \mathrm{Zn})$ and brass $80(20 \% \mathrm{Zn})$ have been produced by spraying molten metal with water at high pressure in the powder metallurgy plant belonging to the Metallurgical Research Centre (CIME) in Havana. A physical-chemical and technological characterization of the powders has been carried out and the optimum parameters have been determined for the formation and sintering of details from these powders. The mechanical properties of these details can rival bronze and iron in applications such as in household electrical goods and in construction.
\end{abstract}

Keywords: Brass details. Powder metallurgy.

\section{INTRODUCCIÓN}

Una de las aleaciones de cobre más importantes es la aleación $\mathrm{Cu}-\mathrm{Zn}$. En comparación con el cobre, el latón tiene mejores propiedades mecánicas y más resistencia a la corrosión.

En la figura 1 se muestra el diagrama de equilibrio $\mathrm{Cu}-\mathrm{Zn}$. Se puede observar que los latones, según su estructura, se pueden clasificar en tres grupos principales: $\alpha, \beta$ y $\alpha+\beta$. El latón $\alpha$ contiene hasta un $39 \% \mathrm{Zn}$, alcanzando la plasticidad máxima con el $30 \% \mathrm{Zn}$. Con este material se puede realizar el estampado profundo y el tratamiento por

$(\bullet)$ Trabajo recibido el día 12 de julio de 1995

(*) Centro de Investigaciones Metalúrgicas (CIME). Avda. 51, 23611. 11300. C. Habana (Cuba).

(**) Centro Nacional de Investigaciones Metalúrgicas (CSIC). Avda. de Gregorio del Amo, 8. 28040-Madrid (España). corte (1 y 2). Los latones $\beta$ no admiten una deformación plástica en frío elevada, pero a temperaturas altas, se hacen muy plásticos y se les puede aplicar la forja y laminación. Las propiedades mecánicas de los latones $\alpha+\beta$ son intermedias de los latones $\alpha$ у $\beta$ (3).

La calidad de las piezas de latón obtenidas por pulvimetalurgia, se determina por la composición granulométrica y química y por la estructura y grado de oxidación del polvo. Para la selección de la composición química del polvo hay que tener en cuenta, además del costo comparativo de cobre y cinc, la influencia del contenido de cinc en el comportamiento del polvo durante el prensado, la tendencia a la formación de defectos durante la sinterización de las piezas y las propiedades mecánicas de los latones (4).

El oxígeno, en forma de óxido de cinc, no se elimina durante la sinterización y afecta de forma negativa a las propiedades mecánicas de las piezas y disminuye la resistencia a la tracción y el grado de 


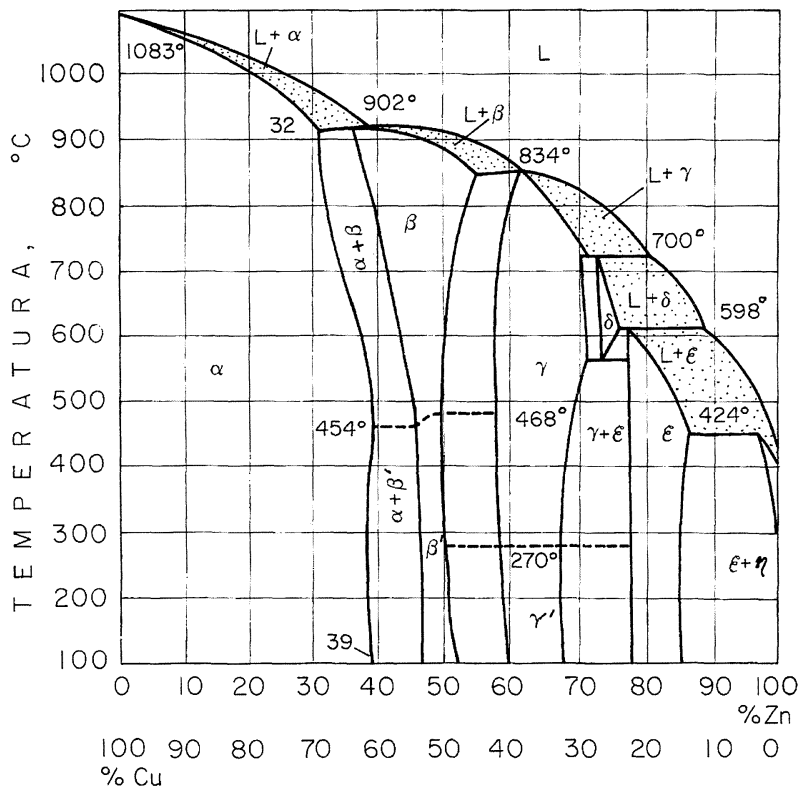

FIG. 1.- Diagrama de equilibrio $\mathrm{Cu}-\mathrm{Zn}$.

FIG. 1.- Diagram of Cu-Zn equilibrium.

plasticidad (5). La práctica industrial de fabricación de piezas de latón demuestra que la estabilidad de las propiedades mecánicas de las piezas se alcanza con un polvo que contiene entre $0,08-0,23 \% \mathrm{O}_{2}(6)$.

La temperatura de sinterización de las piezas de latón puede oscilar entre 800 y $900{ }^{\circ} \mathrm{C}$, y el tiempo entre 60 y $110 \mathrm{~min}$. El aumento de la temperatura de sinterización del latón $60(40 \% \mathrm{Zn})$ por encima de $850{ }^{\circ} \mathrm{C}$ provoca la aparición de la fase líquida, mientras que para el latón 80 ( $20 \%$ Zn) la temperatura puede aumentar por encima de $850^{\circ} \mathrm{C}$ sin que surja la fase líquida (4, 6 y 7). Esta diferencia se debe a la alta sensibilidad de la aleación $\mathrm{Cu}-\mathrm{Zn}$ a las condiciones de sinterización, ya que el cinc tiene tendencia a la evaporación y oxidación.

En este trabajo se determinan los parámetros óptimos para conformar y sinterizar las piezas a partir de polvo de latón 80 y de latón 60 producidas por pulvimetalurgia en la planta del CIME. Los. polvos se producen mediante atomización del metal fundido con agua a alta presión, que garantiza la estabilidad del contenido de $\mathrm{O}_{2}$ en las partículas de diferente granulometría (8).

\section{PARTE EXPERIMENTAL}

\subsection{Obtención del polvo de latón}

El polvo de latón se obtiene mediante la atomización de la aleación fundida, con agua a alta presión. En la figura 2 se muestra el diagrama del proceso y en la figura 3 el esquema de la instalación de pulverización.

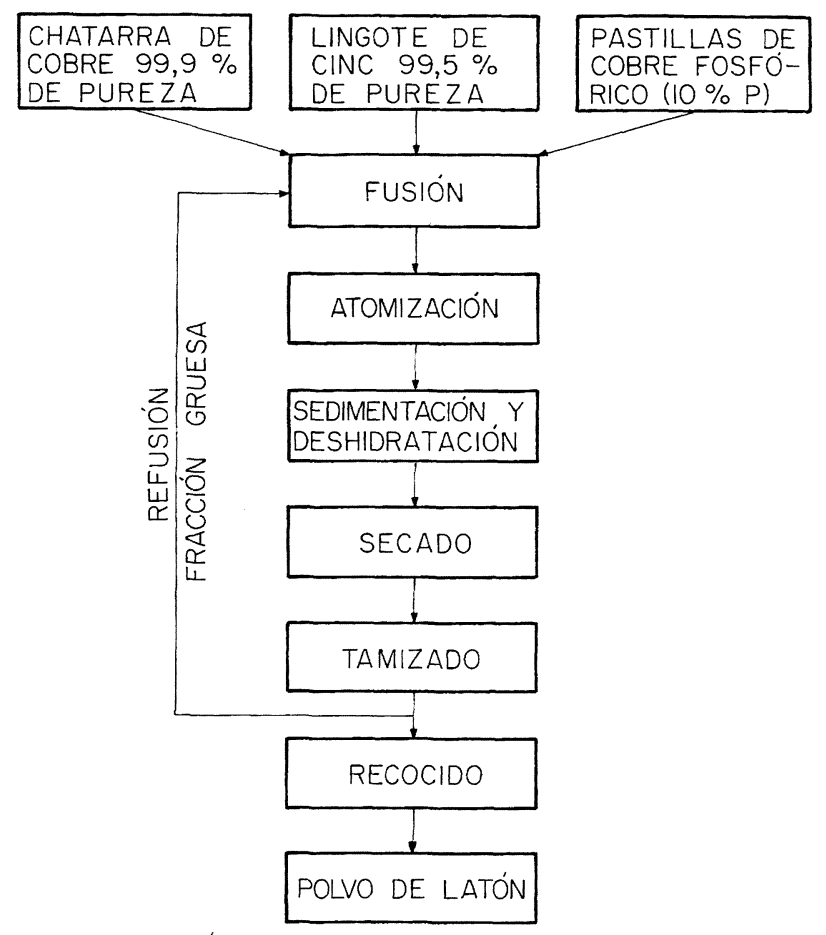

FIG. 2.- Esquema general del proceso.

FIG. 2.- General layout of the process.

Como materia prima, se emplea chatarra de cobre con $99,9 \%$ de pureza y lingote de cinc con $99,5 \%$ de pureza. La fusión se realiza en un horno de inducción con una capacidad de $100 \mathrm{~kg}$ de metal por colada. Primero se añade al horno cobre que, una vez fundido, se desoxida con la adición al baño de pastillas de cobre fosfórico $(10 \% \mathrm{P})$. A continuación, se agrega el lingote de cinc al baño de cobre fundido y se mantiene la temperatura del baño a $1.150-1.200{ }^{\circ} \mathrm{C}$ durante $10 \mathrm{~min}$ para su homogeneización. La cantidad de cobre y cinc se calcula según el tipo de latón que se desea obtener.

La atomización del metal líquido se realiza con agua a una presión de $10 \mathrm{MPa}$ y con un diámetro de flujo del metal de $10 \mathrm{~mm}$.

Después de la deshidratación con aire caliente, el polvo, con una humedad entre 8 y $10 \%$, se seca en un horno a $80^{\circ} \mathrm{C}$.

El polvo se tamiza y luego se somete a un recocido en una corriente de gas reductor para preparar las propiedades químicas y tecnológicas.

\subsection{Caracterización}

Se han obtenido dos tipos de polvo de latón: con $80 \% \mathrm{Cu}$ y $20 \% \mathrm{Zn}$ (latón 80 ) y con $60 \% \mathrm{Cu}$ y 40 $\%$ Zn (latón 60).

En la tabla I se ofrece la composición granulométrica de los polvos de latón, determinada según la Norma ISO 4497. 


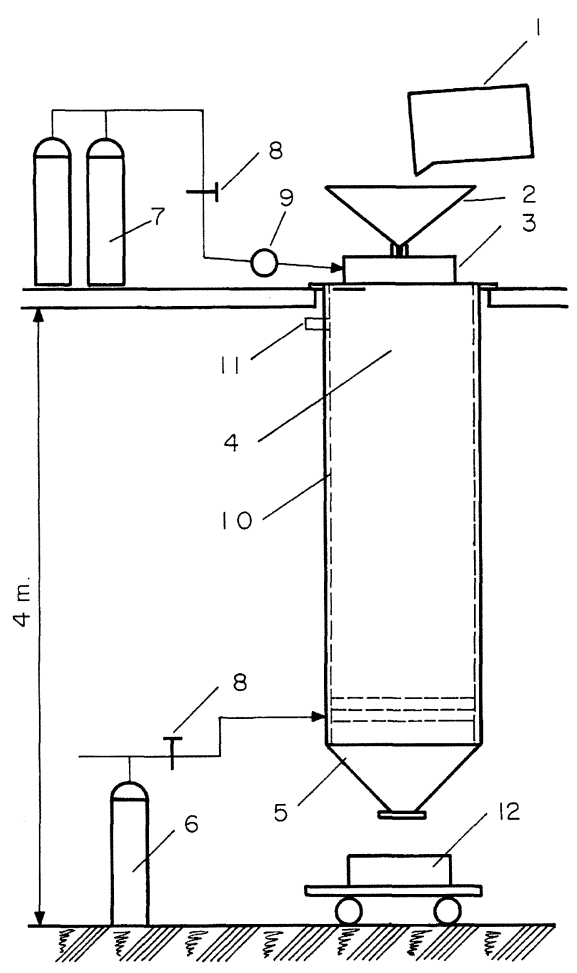

FIG. 3.- Instalación de pulverización (1: Horno de inducción, 2: Metal líquido, 3: Inyector, 4: Cámara de pulverización, 5: Colector de polvo, 6: Gas $\mathrm{N}_{2}$ para enfriamiento, 7: Gas $\mathrm{N}_{2}$ para pulverización, 8: Regulador de presión, 9: Manómetro, 10: Camisa de agua, 11: Extractor, y 12: Depósito de acero para recoger el polvo).

FIG. 3.- Powdering plant (1: Induction furnace, 2: Liquid metal, 3: Injector, 4: Powdering chamber; 5: Powder collector, 6: Gas $\mathrm{N}_{2}$ for cooling, 7: Gas $\mathrm{N}_{2}$ for powdering, 8: Pressure regulator, 9: Manometer, 10: Water jacket, 11: Extractor, 12: Steel storage tank for powder).

En la tabla II se ofrece, para los polvos de latón, la composición química, densidad aparente determinada según la Norma ISO 3923/1 y 3923/2, y fluidez según la Norma ISO 4490.

La tabla III muestra los valores del ensayo de compresibilidad de los polvos de latón, realizado

TABla I.- Composición granulométrica de los polvos de latón, \% en masa

TABLE I.- Granulometric composition of the brass powders, \% in mass

\begin{tabular}{|c|c|c|}
\hline Fracción, $\mu \mathrm{m}$ & Latón 80 & Latón 60 \\
\hline $160-125$ & 11,08 & 4,18 \\
$125-100$ & 10,36 & 8,76 \\
$100-80$ & 7,26 & 12,50 \\
$80-40$ & 65,21 & 57,75 \\
$<40$ & 6,12 & 16,81 \\
\hline
\end{tabular}

TABLA II.- Características de los polvos de latón

TABLE II.-Characteristics of the brass powders

\begin{tabular}{|c|c|c|c|c|c|c|}
\hline \multirow{2}{*}{ Latón } & \multicolumn{4}{|c|}{ Composición química, \% en masa } & \multirow{2}{*}{$\begin{array}{c}\text { Densidad } \\
\text { aparente, } \\
\mathrm{g} / \mathrm{cm}^{3}\end{array}$} & \multirow{2}{*}{$\begin{array}{c}\text { Fluidez } \\
\mathrm{s} / 50, \mathrm{~g}\end{array}$} \\
\hline & $\mathrm{Zn}$ & $\mathrm{Cu}$ & $\mathrm{Pb}$ & $\mathrm{Sn}$ & & \\
\hline $\begin{array}{c}\text { Latón } \\
80\end{array}$ & $20 \pm 0,5$ & $80 \pm 0,5$ & 0,20 & 0,32 & 3,60 & 34 \\
\hline $\begin{array}{c}\text { Latón } \\
60\end{array}$ & $39,5 \pm 0,5$ & $60 \pm 0,5$ & 0,20 & 0,32 & 3,09 & 47 \\
\hline
\end{tabular}

según norma ISO 3927, para determinar la capacidad que tienen los polvos de asimilar la presión durante el prensado y, valorando cuantitativamente este ensayo, se determina la densidad y porosidad de los compactos en verde y sinterizados. La temperatura de sinterización es de $880{ }^{\circ} \mathrm{C}$ para el polvo de latón 80 y $830^{\circ} \mathrm{C}$ para el de latón 60 . El tiempo de retención a la temperatura de sinterización es de 60 min para ambos polvos.

\section{RESULTADOS}

El comportamiento del polvo de latón durante el procesamiento, así como las propiedades del producto terminado, dependen en gran parte de las características básicas del material en polvo. Aparte de la composición química y pureza, las características básicas de un polvo de metal son el tamaño de la partícula y la distribución del tamaño, la forma de la partícula, la densidad aparente y la microestructura de la partícula.

La densidad del compactado verde es una de las propiedades más importantes, ya que indica la efectividad del compactado y determina también el comportamiento del material durante el sinterizado subsiguiente. En la tabla III se puede observar que se ha obtenido una buena densidad para los compactados en verde de los latones, en comparación con los sinterizados.

El proceso de sinterización se efectúa generalmente a una temperatura inferior a la del constituyente de más alto punto de fusión. La sinterización es, esencialmente, un proceso de enlace de cuerpos sólidos por fuerzas atómicas, y las temperaturas elevadas suelen ser favorables para la sinterización.

Una vez realizada la sinterización con los polvos de latón obtenidos, con las piezas fabricadas con los sinterizados se procede al estudio de la estructura y de las propiedades mecánicas.

\subsection{Sinterización del latón 80}

En la fabricación de estos sinterizados se elaboró en el diseño experimental un modelo estadístico 
TABLA III.- Compresibilidad de los polvos de latón

TABLE III.-Compressibility of the brass powders

\begin{tabular}{|c|c|c|c|c|c|c|c|c|}
\hline \multirow{2}{*}{$\begin{array}{c}\text { Presión } \\
\text { específica } \\
\mathrm{MPa}\end{array}$} & \multicolumn{3}{|c|}{\begin{tabular}{c} 
Compactos en verde \\
\cline { 2 - 8 }
\end{tabular}} & \multicolumn{2}{|c|}{$\begin{array}{c}\text { Densidad, } \\
\mathrm{g} / \mathrm{cm}^{3}\end{array}$} & \multicolumn{2}{c|}{$\begin{array}{c}\text { Porosidad, } \\
\%\end{array}$} & \multicolumn{2}{c|}{$\begin{array}{c}\text { Densidad, } \\
\mathrm{g} / \mathrm{cm}^{3}\end{array}$} & \multicolumn{2}{c|}{$\begin{array}{c}\text { Porosidad, } \\
\%\end{array}$} \\
\cline { 2 - 9 } & $\begin{array}{c}\text { Latón } \\
80\end{array}$ & $\begin{array}{c}\text { Latón } \\
\text { Latón }\end{array}$ & $\begin{array}{c}\text { Latón } \\
80\end{array}$ & 60 & $\begin{array}{c}\text { Latón } \\
80\end{array}$ & $\begin{array}{c}\text { Latón } \\
60\end{array}$ & $\begin{array}{c}\text { Latón } \\
80\end{array}$ & $\begin{array}{c}\text { Latón } \\
60\end{array}$ \\
\hline 200 & 6,60 & 6,48 & 22,12 & 20,10 & 6,98 & 6,78 & 18,41 & 17,30 \\
400 & 6,82 & 6,70 & 19,49 & 18,07 & 7,26 & 6,90 & 15,22 & 14,85 \\
500 & 7,04 & 7,03 & 16,92 & 13,42 & 7,29 & 7,06 & 14,79 & 13,05 \\
600 & 7,24 & 7,22 & 14,78 & 11,08 & 7,41 & 7,28 & 13,73 & 10,34 \\
800 & 8,11 & 7,23 & 14,29 & 10,96 & 8,37 & 7,36 & 12,22 & 9,36 \\
\hline
\end{tabular}

factorial $2^{3}$, siendo dos el número de niveles (alto y bajo) y tres el número de variables de operación (presión específica de prensado, temperatura y tiempo de sinterización).

\section{Nivel bajo Nivel alto}

$(-) \quad(+)$

$X_{1}$ Presión específica, $\mathrm{MPa}$

$500 \quad 700$

$X_{2}$ Temperatura de sinterización, ${ }^{\circ} \mathrm{C}$

800

880

$X_{3}$ Tiempo de sinteriza-

ción, min

En la tabla IV se presentan los valores obtenidos en los ensayos realizados, según esta matriz de diseño, de la compresión radial, flexión y dureza del latón, determinados según las Normas ISO 2739, ISO 3325 e ISO 4498/1. De estos resultados se puede estáblecer que los valores óptimos de los parámetros tecnológicos para la fabricación del latón 80 son los siguientes:

- Presión específica: $600 \mathrm{MPa}$

- Temperatura de sinterización: $880{ }^{\circ} \mathrm{C}$

- Tiempo de sinterización: 60 min

En la figura 4 se pueden ver bujes y arandelas fabricadas con latón 80 y latón 60 , respectivamente. El espesor de las arandelas es de $6 \mathrm{~mm}$.

\subsection{Sinterización del latón 60}

Para el latón 60 se realiza un diseño experimental análogo al del latón 80.

En la tabla V se ofrecen las condiciones de ensayo y los valores de la compresión radial y dureza.

TABLA IV.- Sinterizado del latón 80

TABLE IV.- Sintering of brass 80

\begin{tabular}{|c|c|c|c|c|c|c|}
\hline \multirow{2}{*}{ Ensayo } & \multicolumn{3}{|c|}{ Condiciones del ensayo } & \multicolumn{3}{c|}{ Propiedades mecánicas } \\
\cline { 2 - 7 } & $\begin{array}{c}\text { Presión, } \\
\text { MPa }\end{array}$ & $\begin{array}{c}\text { Temperatura, } \\
{ }^{\circ} \text { C }\end{array}$ & $\begin{array}{c}\text { Tiempo, } \\
\text { min }\end{array}$ & $\begin{array}{c}\text { Compresión, } \\
\text { radial, MPa }\end{array}$ & $\begin{array}{c}\text { Flexión, } \\
\text { MPa }\end{array}$ & $\begin{array}{c}\text { Dureza, } \\
\text { HB }\end{array}$ \\
\hline 1 & 500 & 800 & 30 & 81 & 44 & 48 \\
2 & 700 & 800 & 30 & 147 & 35 & 53 \\
3 & 500 & 880 & 30 & 462 & 77 & 47 \\
4 & 700 & 880 & 30 & 500 & 90 & 55 \\
5 & 500 & 800 & 90 & 237 & 77 & 51 \\
6 & 700 & 800 & 90 & 302 & 86 & 58 \\
7 & 500 & 880 & 90 & 257 & 68 & 47 \\
8 & 700 & 880 & 90 & 276 & 105 & 55 \\
\hline
\end{tabular}




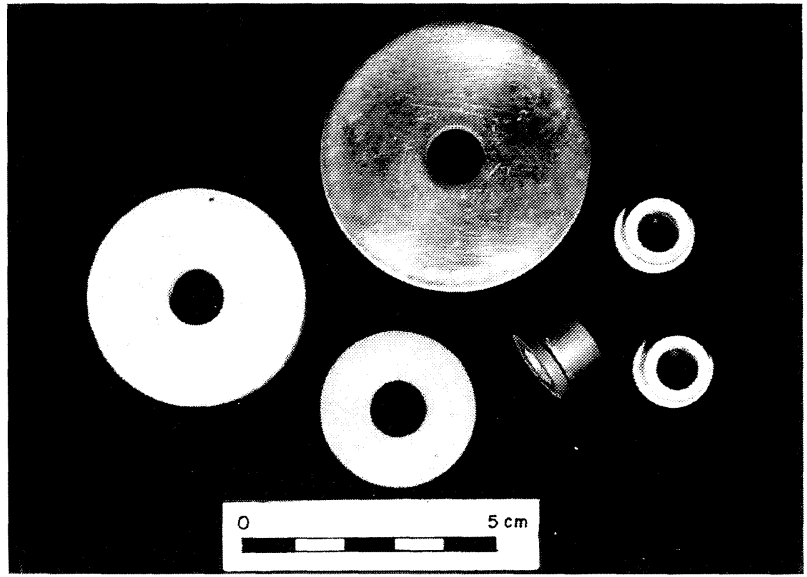

FIg. 4.-Bujes de latón 80 y arandelas de latón 60 .

FIG. 4.-Boss of brass 80 and washers of brass 60.

TABLA V.- Sinterizado de latón 60

TABLE V.- Sintering of brass 60

\begin{tabular}{|c|c|c|c|c|c|}
\hline Ensayo & \multicolumn{2}{|c|}{ Condiciones del ensayo } & \multicolumn{2}{c|}{$\begin{array}{c}\text { Propiedades } \\
\text { mecánicas }\end{array}$} \\
\hline & $\begin{array}{c}\text { Presión, } \\
\text { MPa }\end{array}$ & $\begin{array}{c}\text { Temperatura, } \\
{ }^{\circ} \text { C }\end{array}$ & $\begin{array}{c}\text { Tiempo, } \\
\text { min }\end{array}$ & $\begin{array}{c}\text { Compresión } \\
\text { radial, } \\
\text { MPa }\end{array}$ & $\begin{array}{c}\text { Dureza } \\
\text { HB }\end{array}$ \\
\hline 1 & 400 & 810 & 30 & 110 & 29 \\
2 & 700 & 810 & 30 & 231 & 33 \\
3 & 400 & 850 & 30 & 188 & 29 \\
4 & 700 & 850 & 30 & 259 & 35 \\
5 & 400 & 810 & 90 & 223 & 38 \\
6 & 700 & 810 & 90 & 304 & 45 \\
7 & 400 & 850 & 90 & 195 & 32 \\
8 & 700 & 850 & 90 & 377 & 42 \\
\hline
\end{tabular}

De los resultados obtenidos se deduce que los valores óptimos de los parámetros para la fabricación del latón 60 son:

- Presión especifica: $600 \mathrm{MPa}$

- Temperatura de sinterización: $830{ }^{\circ} \mathrm{C}$

- Tiempo de sinterización: 90 min

\subsection{Análisis por microsonda de los latones}

Se ha estudiado la estructura de los latones en un microscopio electrónico de barrido con microsonda JEOL modelo JSM-840, dotado con un analizador LINK AN-10000.

En la tabla VI se ofrece el análisis de cada latón, siendo el cobre y el cinc los únicos elementos detectados en el espectro realizado. Los valores son la media de tres análisis realizados en tres zonas de la muestra, por ser estos valores muy próximos.

La figura 5 corresponde a una zona de la sección longitudinal de un buje de latón 80 , donde se puede observar una estructura monofásica compuesta por
TABLA VI.- Análisis químico puntual de los latones, $\%$ en masa

TABLE VI.- Point chemical analysis of the brasses, $\%$ in mass

\begin{tabular}{|c|c|c|}
\hline Latón & $\mathrm{Cu}$ & $\mathrm{Zn}$ \\
\hline 80 & 81,34 & 18,84 \\
\hline 60 & 58,97 & 43,11 \\
\hline
\end{tabular}

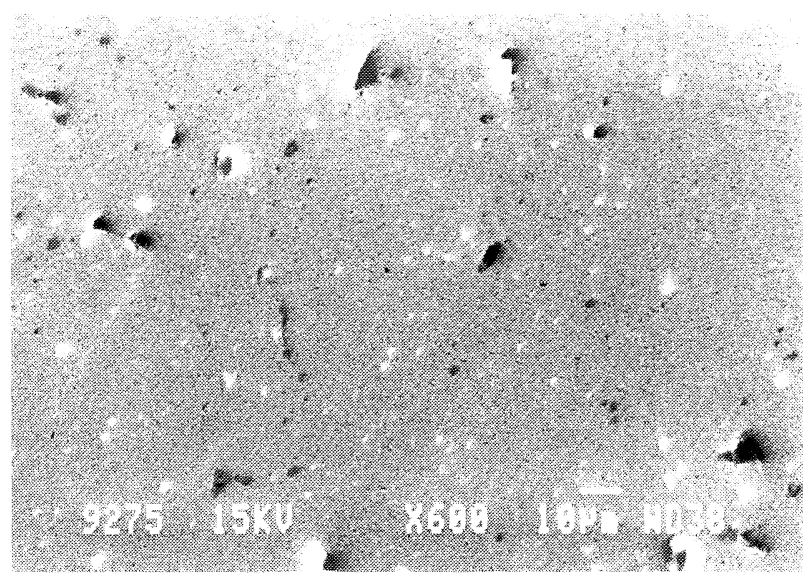

FIG. 5.- Estructura de latón $80 . \times 600$.

FIG. 5.- Structure of brass $80 . \times 600$.

la disolución sólida $\alpha$, con presencia de alguna porosidad.

La figura 6 corresponde a una zona del borde de la sección transversal de una arandela de latón 60 . En la figura 7 se muestra un detalle de una zona localizada en la figura anterior, donde se puede apreciar una estructura monofásica $\alpha$ de granos redondeados y presencia de porosidad abundante. En la figura 8 se observa una zona del centro de la

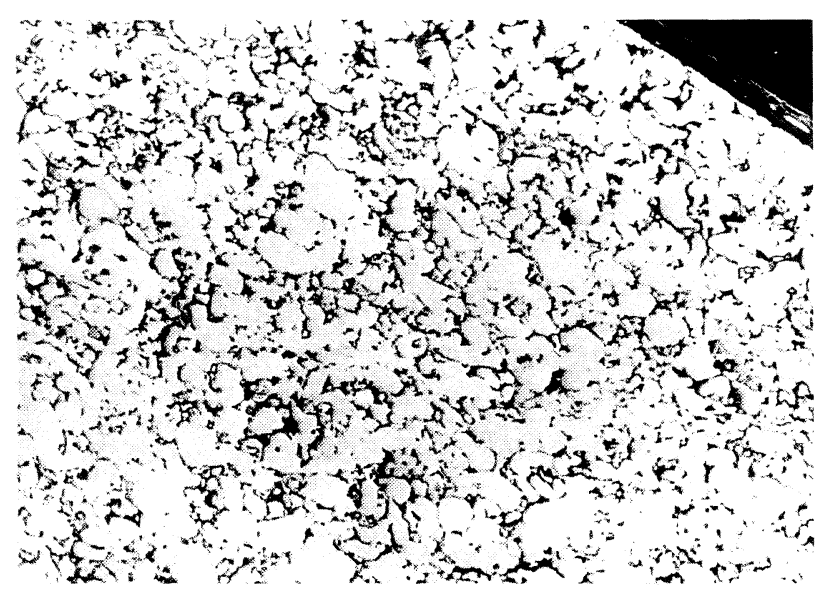

Fig. 6.- Borde de la sección transversal de una arandela de latón $60 . \times 100$.

FIG. 6.- Edge of the transversal section of a washer of brass $60 . \times 100$. 


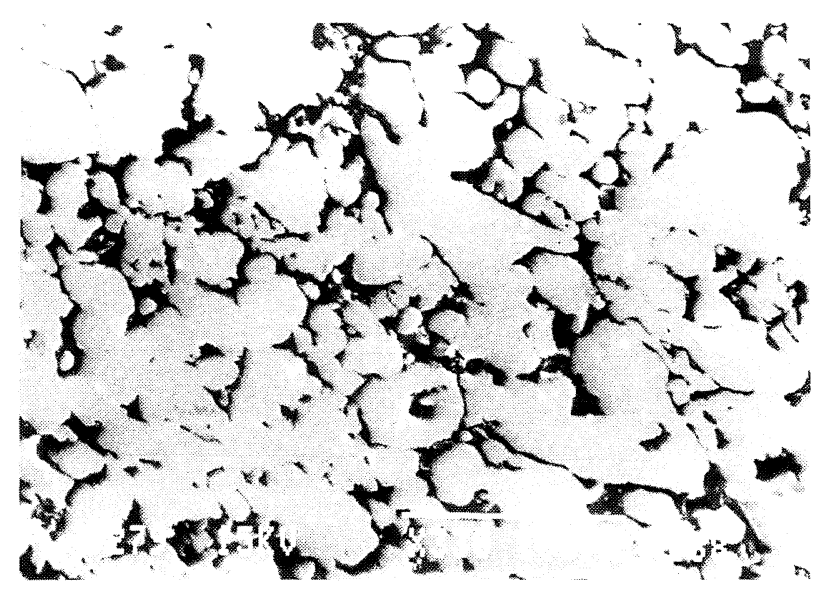

Fig. 7.- Zona en detalle localizada en la figura 6. Presencia de granos redondeados y porosidad. $\times 300$.

FIG. 7.- Detail of zone from figure 6. Presence of rounded grains and porosity. $\times 300$.

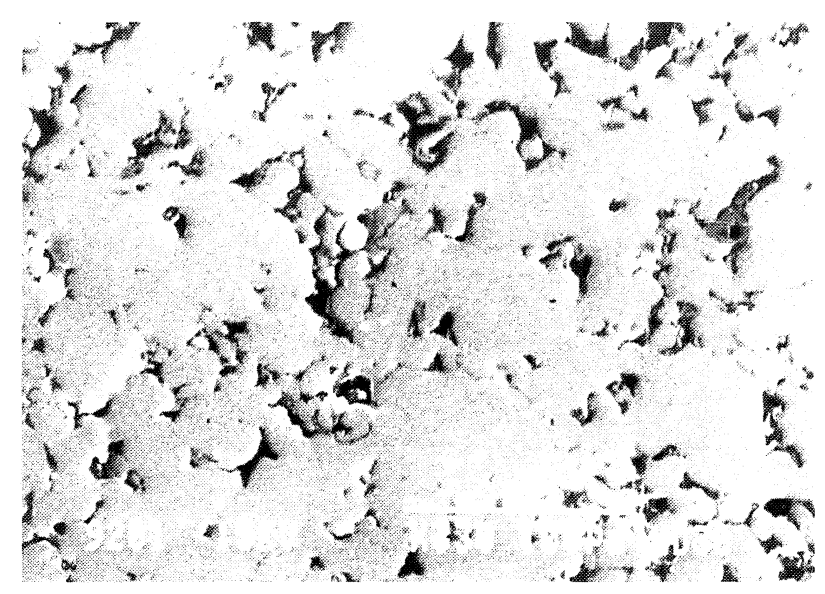

Fig. 8. - Zona del centro de la sección transversal de una arandela de latón $60 . \times 330$.

FIG. 8.- Central zone of the transversal section of a washer of brass $60 . \times 330$.

sección transversal de la arandela, más densa que la estructura del borde.

\subsection{Propiedades mecánicas de los latones}

En la tabla VII se ofrecen los valores de las propiedades mecánicas de los latones, y se comparan con los valores para el bronce con $10 \%$ de Sn y para el hierro atomizado. Se puede observar que los latones pueden competir con éxito en el campo de los materiales de construcción (9).

El latón 80 se utiliza en la fabricación de bujes para la puerta de refrigeradores con buenos resultados. El latón 60 se utiliza en la fabricación de arandelas que, después de pulidas y barnizadas, mantienen un buen estado superficial y tienen aplicación en la construcción de lámparas.
TABLA VII.- Propiedades mecánicas del latón, del bronce y del hierro

TABLE VII.- Mechanical properties of brass, bronze and iron

\begin{tabular}{|c|c|c|c|}
\hline Material & $\begin{array}{c}\text { Compresión } \\
\text { radial, MPa }\end{array}$ & $\begin{array}{c}\text { Flexión, } \\
\text { MPa }\end{array}$ & $\begin{array}{c}\text { Dureza, } \\
\text { HB }\end{array}$ \\
\hline Latón 80 & $300-400$ & $80-100$ & $45-55$ \\
Latón 60 & $200-400$ & $270-400$ & $40-50$ \\
Bronce & $300-400$ & $265-350$ & $40-50$ \\
Hierro & $300-500$ & $280-290$ & $50-70$ \\
\hline
\end{tabular}

\section{CONCLUSIONES}

Se han optimizado las condiciones de prensado y sinterización en la piezas de latón $80(80 \% \mathrm{Cu}$, $20 \%$ Zn) y latón $60(60 \% \mathrm{Cu}, 40 \% \mathrm{Zn})$, obtenidas a partir de polvos producidos por pulvimetalurgia.

Los parámetros óptimos para la fabricación de las piezas de latón 80 son: presión específica de prensado: $600 \mathrm{MPa}$, temperatura de sinterización: $880{ }^{\circ} \mathrm{C}$ y tiempo de sinterización: $60 \mathrm{~min}$. Estos parámetros para el latón 60 son: presión específica de prensado: $600 \mathrm{MPa}$, temperatura de sinterización: $830{ }^{\circ} \mathrm{C}$ y tiempo de sinterización: $90 \mathrm{~min}$.

Ambos materiales poseen buenas propiedades mecánicas y pueden utilizarse ampliamente en las piezas de construcción.

\section{REFERENCIAS}

(1) Guliaev, A.P. Metalografía. 5ª Ed. Moscú. Metallurgiya, 1977: 606-610.

(2) Kozlov, Yu. Ciencia de los materiales. Ed. MIR. Moscú, 1986: 115-119.

(3) Calvo Rodes, F. Prontuario Metalotécnico. Tomo 1. Ed. INTA. Madrid, 1963.

(4) Academia de Ciencias de Ucrania. Materiales a partir de polvos metálicos. Materiales de construcción con base de polvo de latón. Kiev, 1983: 33-42.

(5) Rodomyselsky, I.D., Gaiduchenko, G.K., Kalinyuk, N.N., Medvedovsky, A.B., Nichiporenko,O.S. y Chaikina, N.G. Poroshk. Metall., (7), 1983: 1-4.

(6) Dovydenkova, A.V., Sergeev, V.L., Chernov, V.A. y Kiseleva, E.V. Poroshk. Metall., (4), 1990: 44-47.

(7) Dovydenkova, A.V., Sergeev, V.L., Chernov, V.A., Kuznetsova, Z.V. y Kiseleva, E.V. Poroshk. Metall., (9) 1988: 96-98.

(8) Belyakov, V.A., Medvedovsky, A.B., Nichiporenko, O.S., Poteshrina, V.P., Naida, Yu.I. y Potasheva, G.A. Poroshk. Metall., (6), 1981: 13-15.

(9) Academia de Ciencias de Ucrania. Materiales a partir de polvos metálicos. Perspectivas de la pulvimetalurgia en la producción de piezas para instalaciones técnico-sanitarias. Kiev, 1980. 